\title{
Virtual Screening of Cablin Patchouli Herb as a Treatment for Heat Stress: A Study Based on Network Pharmacology, Molecular Docking, and Experimental Verification
}

\author{
Yan Xu, ${ }^{1}$ Lizhong Ding, ${ }^{2}$ Zhongtian Wang, ${ }^{1}$ Yanbo Wang $\left(\mathbb{D},{ }^{1}\right.$ and Liping Sun $\mathbb{D}^{1}$ \\ ${ }^{1}$ Changchun University of Chinese Medicine, Changchun, 130117, China \\ ${ }^{2}$ Affiliated Hospital, Changchun University of Chinese Medicine, Changchun, Jilin 130021, China
}

Correspondence should be addressed to Yanbo Wang; 14463798@qq.com and Liping Sun; slpcczyydx@sina.com

Received 11 July 2020; Revised 21 February 2021; Accepted 26 February 2021; Published 10 March 2021

Academic Editor: Hilal Zaid

Copyright (c) 2021 Yan Xu et al. This is an open access article distributed under the Creative Commons Attribution License, which permits unrestricted use, distribution, and reproduction in any medium, provided the original work is properly cited.

\begin{abstract}
Heat-related diseases have long been known to damage the structure and function of essential macromolecules such as proteins, lipids, and nucleic acids, thereby compromising the integrity of cells and tissues and the physiological functions of the entire organism. Heat stress is the physical discomfort caused by overheating the body and is also the initial manifestation of heat-related diseases. Cablin patchouli herb (CPB) has been used in China for two thousand years and has been used to treat heat stress, but to date, no related mechanistic research is available. In this study, KEGG and PPI networks and the TCMSP and GEO databases were used to explore the components of CPB in relation to heat stress: quercetin, genkwanin, irisolidone, 3,23-dihydroxy-12-oleanen28-oic acid, and quercetin 7-O- $\beta$-D-glucoside. The targets identified were EGFR, NCOA1, FOS, HIF1A, NFKBIA, and NCOA2; these proteins were verified by molecular docking and experimental verification. In short, our research represents the first report on the use of the traditional Chinese medicine $\mathrm{CPB}$ to treat heat stress and thus has pioneering significance.
\end{abstract}

\section{Introduction}

As global warming intensifies, heat-related diseases have become a major public health issue during heat waves around the world, even in areas traditionally considered to be temperate and cold. In July 1995, a heat wave in the United States killed more than 700 people in less than 7 days, and more than 3,000 people visited the emergency room [1]. In 2003 and 2010, two severe heat waves affected Western Europe and Russia, respectively, causing 70,000 deaths and 55,000 deaths $[2,3]$. More seriously, children account for almost half of the total population suffering from heat-related diseases $(47.6 \%)$ [4].

Heat-related diseases have been known since ancient times; they reflect the relationship between the human body and heat imbalance [5], and long-term exposure to excessive heat or certain drugs that cause fever are the main drivers of heat-related diseases [6]. Heat can damage the structure and function of essential macromolecules such as proteins, lipids, and nucleic acids, thereby compromising the integrity of cells and tissues and the physiological functions of the entire organism, especially the central nervous system [7-10]. Heat-related conditions can be categorized into heat rash, heat cramps, heat stress, heat exhaustion, and heat stroke according to their severity; all these conditions have fever in common $[4,6]$. In this study, we focused on exploring heat stress, an early manifestation of heat-related diseases. The symptoms of heat stress include prolonged fever, excess sweating or thirst, tiredness, and cramps; however, tachycardia and nervous system damage do not manifest.

Cablin patchouli herb (CPB) was first published in "Mingyi Bielu," a medical book of the Han Dynasty, more than 2,000 years ago. It has the effect of "dispelling heat and dampness" and is recognized as a cure for heat stress. The Chinese patent medicine Huoxiang Zhengqi water, which uses $\mathrm{CPB}$ as its main medicinal material, was described in the textbook "Internal Medicine of Traditional Chinese 
Medicine" in China, and it is also considered to exhibit excellent effects in treating heat stress (yin-related heatrelated diseases). It should be noted that according to the Chinese Yin-Yang Theory, the scope of heat-related diseases is broad and can be divided into Yin and Yang. Heat stress is usually biased towards yin-related heat-related diseases that is relatively less harmful to the human body. Heat exhaustion and heat stroke are generally regarded as yangrelated heat-related diseases that are more harmful to the human body. CPB is usually used to treat heat stress (i.e., yin-related heat-related diseases). At present, reports on $\mathrm{CPB}$ treatment of heat stress are concentrated in the Chinese literature. Fusen used scraping therapy combined with Huoxiang Zhengqi water (for which the main component is $\mathrm{CPB}$ ) to treat 48 cases of yin-related heat stroke and achieved good results [11]. Li used Huoxiang Zhengqi water to treat 240 cases of pediatric fever and found that children's heatrelated symptoms were significantly reduced 3-4 hours after treatment [12]. Gu Fang and Rinan also used Huoxiang Zhengqi water to treat 18 cases of pediatric heat rash, a rash caused by heat. The effective rate for the treatment group reached $94.4 \%$, which was significantly better than that of the control group [13]. Chaobin and Wanzhou elaborated on the theoretical basis of traditional Chinese medicine for the treatment of yin-related heat stroke with CPB. They believed that $\mathrm{CPB}$ can also treat fever and diarrhea [14]. There are some studies on the treatment of diarrhea $[15,16]$. In short, since 2000, Chinese people have reliably used CPB to treat heat-related diseases, and its therapeutic effects are very good. However, with the strong development of science in the modern era, a great deal of interest has arisen in understanding such traditional disease treatment methods. It is necessary to study specific treatment mechanisms and to clarify their specific ingredients and targets.

However, there is a major difficulty in exploring the treatment mechanism of CPB: the complexity of Chinese medicine components and the multiplicity of pharmacological effects make it difficult to conduct comprehensive and systematic research in the context of Chinese medicine [17]. To solve this problem, after decades of research, Li Shao proposed in 1999 that there may be a connection between TCM syndrome and molecular networks [18-23]. Based on the rapid development of systems biology and computer technology, network pharmacology was born. Network pharmacology is based on the herb network-biological network-phenotype network interaction network [20]. Through network analysis, we can systematically observe the interventions and impacts of drugs on diseases and reveal the mysteries of drug molecules synergistically acting on the human body. This is the same as the core concept of traditional Chinese medicine: the principle of multicomponent, multipathway, and multitarget synergistic effects. Network pharmacology is also rapidly becoming a frontier research field in current drug research and next-generation drug research models [18].

Based on the above information, in this study, we used network pharmacology and molecular docking and experimental verification methods to explore the specific mechanisms by which $\mathrm{CPB}$ treats heat stress. Our goal was to enable people outside China to recognize and accept CPB and to help patients with heat-related diseases worldwide.

\section{Materials and Methods}

2.1. The Composition and Targets of CPB. The TCMSP database (http://tcmspw.com/tcmsp.php) [24] was used to identify the active ingredients and targets of CPB. Oral bioavailability $(\mathrm{OB})$, drug-like properties (DL), relative molecular mass (MV), octanol-water partition coefficient (AlogP), number of $\mathrm{H}$-bond coordination electron donors (Hdon), and number of $\mathrm{H}$-bond coordination electron acceptors (Hacc) were used as indicators to compare the physical and chemical properties of the CPB components and to study the similarities and differences between the components.

2.2. Genes Differentially Expressed during Heat Stress. Differentially expressed genes were obtained from NCBIGEO, a free public microarray/gene profile database. GEO accession number GSE90763 includes the transcriptome information of 15 volunteers who were subjected to heat stress. The data before exposure (T0) and at the end of heat exposure (T1) were selected for comparison. These data were analyzed using the GEO2R online tool, and 1,789 differentially expressed genes were identified using $|\log \mathrm{FC}| \geq 1$ and $P$ value $<0.05$ as criteria.

2.3. Disease-Drug-Target Network. After the composition of $\mathrm{CPB}$ was obtained using $\mathrm{OB}>30 \%$ and $\mathrm{DL}>0.18$, the targets of $\mathrm{CPB}$ components were downloaded from the TCMSP database, and the target elements were entered into the UniProt database one by one (https://www.UniProt.org/, currently the most complete nonredundant protein sequence database with the most complete sequence data and the richest annotation information in the world) for correction and conversion to GeneSymbol. Next, differentially expressed genes were used to intersect the corrected $\mathrm{CPB}$ target and were imported into Cytoscape (version: 3.6.1) and used to construct the $\mathrm{CPB}$ ingredient-target-disease network.

2.4. Molecular Docking. The protein data for P-gp (PDB ID : 4XWK), CYP3A4 (PDB ID:3NXU), EGFR (PDB ID : 2ITW), FOS (PDB ID:1A02), HIF1A (PDB ID:1H2M), NCOA1 (PDB ID : 1K4W), NCOA2 (PDB ID : 2ZXZ), and NFKBIA (PDB ID :6Y1J) were downloaded from the PDB database (http://www.rcsb.org/). Then, MGLTools 1.5.6 was used to process the protein data, simulate hydrogenation, calculate the charge, merge the nonpolar hydrogen, and save the results as a pdbqt file. According to each protein's ligand, the active site was defined, the grid box coordinates were set, and the box size was defined as $40 \times 40 \times 40$ grid points; the distance between the small grid points was $0.1 \mathrm{~nm}$. AutoDock Vina 1.1.2 was used to simulate the docking of molecules and proteins. The affinity was less than 0 , indicating that the receptor and ligand can spontaneously bind. 
2.5. PPI Network Enrichment and KEGG Analyses. The protein-protein interaction (PPI) network was analyzed using the Search Tool for the Retrieval of Interacting Genes (STRING, http://string.embl.de/) database (organism: Homo sapiens). Kyoto Encyclopedia of Genes and Genomes (KEGG) pathway enrichment analyses were performed using the clusterProfiler package in $\mathrm{R}$ (3.6.1) software with pvaluecutoff $=0.05$ and qvaluecutoff $=0.05$ [25] .

\section{6. $C P B$ Animal Experiment Verification}

2.6.1. Drugs and Materials. CPB was purchased from Jiangyin Tianjiang Pharmaceutical Co., Ltd. (production batch number: 19086254) and diluted to $10 \mathrm{mg} / \mathrm{mL}, 20 \mathrm{mg} /$ $\mathrm{mL}$, and $40 \mathrm{mg} / \mathrm{mL}$ with normal saline (NS) for use. Ibuprofen suspension (production batch number: H19991011) was purchased from Shanghai Johnson Pharmaceutical Co., Ltd. and was used at $0.6 \mathrm{~g} / \mathrm{kg}$ and diluted with NS. The NCOA1 (MD696513) kit, RIPA lysis buffer (article number: MD912016), BCA protein concentration determination kit (article number: MD913053), SDS-PAGE precast gel kit (article number: MD911919), and Rabbit Anti-Mouse antibody (MD912566) were purchased from Beijing Biotek Biomedical Technology Co., Ltd. PVDF membrane (Millipore, USA, ISEQ00010) and medium protein molecular weight marker (26617) were purchased from Thermo, USA, and EGFR antibody (ab32077) and C-FOS antibody (ab134122) were purchased from Abcam, USA. SDS-PAGE system (U.S. BIO-Rad company, model Mini-PROTEAN), protein wet transfer instrument (U.S. BIO-Rad company, model Mini Trans-Blot), and western blot imaging system (U.S. BIO-Rad company, model 170-8280) were employed.

2.6.2. Animals and Models. Forty-two specific pathogenfree (SPF) rats (female, 3-5 weeks old, 50-70 g) were purchased from Liaoning Changsheng Biotechnology Co., Ltd. (experimental animal production license number: NO.SCXK (Liao) 2020-0001) and subjected to the following conditions: rearing temperature- $(24 \pm 3)^{\circ} \mathrm{C}$, relative humidity- $(40 \pm 5) \%$, illumination-alternating light and dark, noise $<55 \mathrm{Db}$, free water and food, and twice-weekly litter change. The animal experimentation was approved by the Animal Ethics Committee of Changchun University of Traditional Chinese Medicine (No. 20190116). The basal body temperature of SD rats was measured 3 days before the experiment, and the average value of the rectal temperature measured at three time points in the morning, midnight, and evening was used as the basal body temperature of the experimental animal. The rats were divided into 7 groups, 6 in each group, and fasted 6 hours before the experiment. Except for the blank group and the blank + NS group, the other 5 groups were subcutaneously injected with 10\% dry yeast suspension $(10 \mathrm{~mL} / \mathrm{kg})$ on the backs of rats. Body temperature was measured every 1 hour after modeling. After successful modeling $\left(\triangle T>0.8^{\circ} \mathrm{C}\right.$, approximately 5 hours), each group was given intragastric administration: the blank + NS and model group were given $31 \mathrm{~mL} / \mathrm{kgNS}$, and the CPB treatment group and the ibuprofen group were given different concentrations of $\mathrm{CPB}$ and $31 \mathrm{~mL} / \mathrm{kg}$ ibuprofen. After the administration, the body temperature was measured every $1 \mathrm{~h}$, a total of 10 times, and the body temperature change value was calculated. Ten hours after the dry yeast group was modeled, a microcapillary was used to collect blood from the fundus venous plexus of the rats in each group. After standing at room temperature for 30 minutes, the blood samples were centrifuged at $4^{\circ} \mathrm{C}$ and 300 $\mathrm{r} / \mathrm{min}$ for 10 minutes, the upper serum was drawn and stored in a $-80^{\circ} \mathrm{C}$ refrigerator for use, and IL- $1 \beta$, IL- 6 , TNF- $\alpha$, and NCOA1 were detected in serum according to the instructions of the ELISA kit. After blood collection, the rats were anesthetized by intraperitoneal injection of $10 \%$ chloral hydrate $(4 \mathrm{~mL} / \mathrm{kg})$. The skin was then cut along the costal arches on both sides, the abdominal cavity was opened, and curved scissors were used to create a small cut at the junction of the diaphragm and the sternum stem; this cut was then extended to both sides, the diaphragm and ribs were cut, the heart was fully exposed, and intravenous drip with a needle was used. The apex of the left ventricle was entered and fixed with a vascular clamp. NS (room temperature) was quickly instilled while cutting the right atrial appendage. When the blood color of the outflow liquid was light and almost clear, the perfusion was stopped. The brain was decapitated immediately, and the hypothalamus was removed from the center of the optic chiasm and gray nodules. The hypothalamus was quickly frozen in liquid nitrogen for 20 minutes and then stored at $-80^{\circ} \mathrm{C}$.

2.6.3. Western Blot Detection. Each experimental group included an equal mass of hypothalamic samples. RIPA buffer was added to produce lysate, and each sample was shaken with a tissue homogenizer for 5 minutes, centrifuged at $12,000 \mathrm{r} / \mathrm{min}$ for 15 minutes to obtain the supernatant, and the protein concentration was measured by BCA. After mixing with the loading buffer, samples were boiled in a boiling water bath for 5 minutes, and SDS-PAGE was employed. The protein was transferred to PVDF membrane by the wet transfer method, and 5\% skimmed milk powder was sealed at room temperature for 2 hours. The primary antibody was incubated overnight at $4^{\circ} \mathrm{C}$. After washing the membrane with TBST, the secondary antibody was added to incubate at $37^{\circ} \mathrm{C}$ for 1 hour. After washing the membrane with TBST, the color developed.

2.7. Statistics. The data were expressed as the mean $\pm \mathrm{SD}$ $(n \geq 3)$ and analyzed by GraphPad Prism 6.0 (GraphPad Software) using Student's $t$-test ( ${ }^{*} p<0.05$ and $\left.{ }^{* *} p<0.01\right)$.

\section{Results}

3.1. General Description of $C P B$. The TCMSP database was used to collect $\mathrm{CPB}$ composition information and produce statistical descriptions based on the OB, DL, MV, AlogP, Hdon, and Hacc parameters, as shown in Table 1. The average value of $\mathrm{OB}$ was 37.90 (20.22), and the average value of DL was $0.20(0.22)$, higher than the value range found by 
TABle 1: Physical and chemical properties of CPB components.

\begin{tabular}{lc}
\hline Index & Result \\
\hline$n$ & 94 \\
OB (mean (SD)) & $37.90(20.22)$ \\
DL (mean (SD)) & $0.20(0.22)$ \\
MW (mean (SD)) & $253.41(118.93)$ \\
AlogP (mean (SD)) & $3.27(1.77)$ \\
Hdon (mean (SD)) & $1.18(2.12)$ \\
Hacc (mean (SD)) & $2.76(3.82)$ \\
\hline
\end{tabular}

The values are expressed as the mean (SD).

previous studies $(\mathrm{OB}>30 \%, \mathrm{DL}>0.18)$ [26]. This shows that $\mathrm{CPB}$ has good oral availability and drug-like properties.

3.2. Further Screening of $C P B$ Components. To eliminate possible false-positive components, $\mathrm{OB}>30 \%$ and $\mathrm{DL}>0.18$ were also used as screening indices [26], and the CPB components were screened more stringently. As shown in Table 2, a total of 11 components satisfied the new screening conditions.

\subsection{Eleven Active Ingredients Docked with P-gp and CYP3A4} Molecules. The absorption, distribution, metabolism, and excretion of CPB in the human body are affected by many factors. Among them, the interaction between P-gp and cytochrome CYP3A4 plays a key role [35]. P-gp is widely distributed and expressed in intestinal epithelial cells, hepatocytes, and capillary endothelial cells and plays an important role in the process by which most heterologous compounds pass through the blood-brain barrier and the blood-testis barrier $[35,36]$. CYP3A4 is the most important metabolic enzyme in the P450 enzyme system and contributes to the metabolism of $1 / 3$ of oral drugs [35, 37]. P-gp and CYP3A4 can synergistically restrict the entry of foreign substances into the human body in the intestine $[35,38]$. To explore whether the active ingredients of CPB can enter the human body and play pharmacological roles, the molecular docking method was used for verification, as shown in Figure 1.

P-gp and CPB molecular docking showed that the 11 active ingredients could successfully dock and that their affinity values were less than 0 . In addition, the docking results of $\mathrm{CYP} 3 \mathrm{~A} 4$ and $\mathrm{CPB}$ with the active ingredient molecules also showed that they could successfully dock, with the exception of acanthoside B. Given this result, acanthoside $\mathrm{B}$ was removed from the list of main active ingredients, and the subsequent steps examined only the remaining ingredients.

3.4. Drug-Ingredient-Target-Disease Network. Genes differentially expressed in heat stress were identified through the GEO database. Using $|\log \mathrm{FC}|>1$ and $P$ value $<0.05$ as criteria, a total of 1,789 differentially expressed genes were obtained (Supplementary Table 1). The CPB component targets were then downloaded from the TCMSP database, and UniProt database correction was performed to remove some targets that have not been manually annotated and reviewed. After correction, only 85 targets with 8 components (Supplementary Table 2) were obtained. Immediately afterwards, the differentially expressed genes of heat-related diseases and the corrected CPB targets were used for intersection, and a drug-component-target-disease network was generated, as shown in Figure 2. Only 5 molecules and their corresponding targets corresponded to heat stress genes. The molecules were quercetin, genkwanin, irisolidone, 3,23-dihydroxy-12-oleanen-28-oic acid, and quercetin $7-\mathrm{O}-\beta$-D-glucoside. Studies have shown that quercetin can increase secretion in cherry tomato fruit in a hot environment and can increase disease resistance [39]. It is worth mentioning that similar to quercetin, those molecules are also found in several other medicinal plants, such as kales, onions, berries, apples, red grapes, broccoli, and cherries as well as tea and red wine [40]. However, the content differs for each medicinal plant. In addition, existing technology cannot make specific judgments about complex chemical reactions that occur simultaneously in different molecules in plants. After a Chinese medicine enters the body, there are also complicated processes of absorption, distribution, metabolism, and excretion. Therefore, we hypothesize that although different Chinese medicines may have some of the same molecules, based on the complex characteristics of multiple components and multiple targets of traditional Chinese medicine, it is still believed that each traditional Chinese medicine, and even each molecule, corresponds with a distinct biological process in the body.

3.5. KEGG Enrichment Analysis. In the previous drugcomponent-target-disease network, we obtained 7 targets for possible treatments: EGFR, FOS, HIF1A, IRF1, NCOA1, NCOA2, and NFKBIA. However, the physiological and metabolic effects of $\mathrm{CPB}$ in the body were still unknown. Therefore, we performed KEGG enrichment analyses, as shown in Figure 3. Notably, in the KEGG enrichment analysis, we found that pathways related to PD-L1 expression and the PD-1 checkpoint pathway in cancer and estrogen signaling pathway were the most enriched.

The occurrence and development of tumors is accompanied by the formation of a special tumor immune microenvironment. Tumor cells can escape immune surveillance and destroy the host's immune checkpoints through various methods, thus avoiding elimination from the host's immune system [41]. Under normal physiological conditions, immune checkpoint molecules are in a balanced state such that the immune response of T cells maintains an appropriate intensity and scope in order to minimize damage to surrounding normal tissues and avoid autoimmune reactions [42]. However, cancer uses a variety of methods to upregulate negative signals through cell surface molecules, thereby inhibiting $\mathrm{T}$ cell activation or inducing apoptosis and promoting cancer progression and metastasis [43]. The immunotherapy method that uses antagonistic antibodies to block the immune checkpoint pathway can release cancer suppression and promote antitumor activity, thereby achieving the purpose of treating cancer. Programmed death 1 (PD-1) and its ligands PD-L1 (B7H1) and 
TABle 2: The 11 main components of CPB.

\begin{tabular}{|c|c|c|c|c|c|c|}
\hline No. & MolID & MolName & OB & $\mathrm{DL}$ & Molecular structure & Reference \\
\hline 1 & MOL002879 & Diop & 43.59 & 0.39 & & {$[27]$} \\
\hline 2 & MOL005573 & Genkwanin & 37.13 & 0.24 & & {$[27]$} \\
\hline 3 & MOL005884 & Patchoulan 1, 12-diol & 38.17 & 0.25 & & {$[27,28]$} \\
\hline 4 & MOL005890 & Pachypodol & 75.06 & 0.4 & & {$[29,30]$} \\
\hline 5 & MOL005911 & 5-Hydroxy-7, 4'-dimethoxyflavanone & 51.54 & 0.27 & & [31] \\
\hline 6 & MOL005916 & Irisolidone & 37.78 & 0.3 & & {$[27]$} \\
\hline 7 & MOL005918 & Phenanthrone & 38.7 & 0.33 & & {$[27,32]$} \\
\hline 8 & MOL005921 & Quercetin 7-O- $\beta$-D-glucoside & 49.57 & 0.27 & & [31] \\
\hline 9 & MOL005922 & Acanthoside B & 43.35 & 0.77 & & [27] \\
\hline
\end{tabular}


TABle 2: Continued.

\begin{tabular}{lllll}
\hline No. MolID & MolName & $\mathrm{OB}$ & $\mathrm{DL}$ & Molecular structure \\
11 & MOL005923 & 3, 23-Dihydroxy-12-oleanen-28-oic acid & 30.86 & 0.86
\end{tabular}

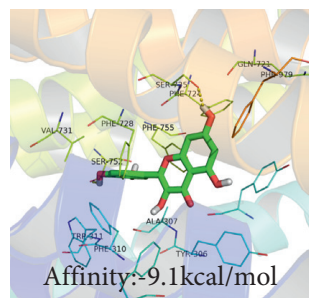

(A1)

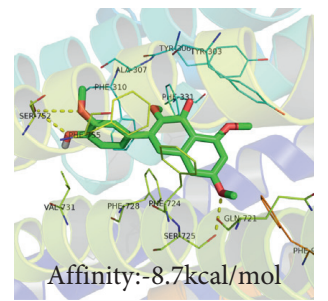

(A5)

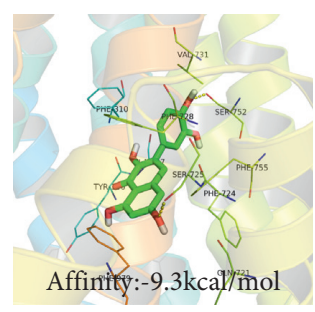

(A9)

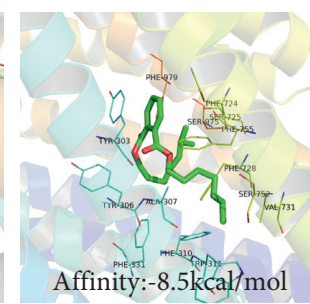

(A2)

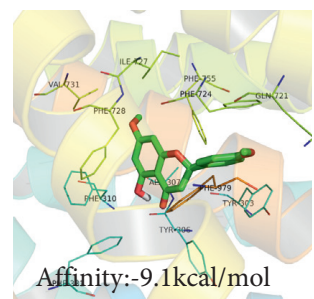

(A6)

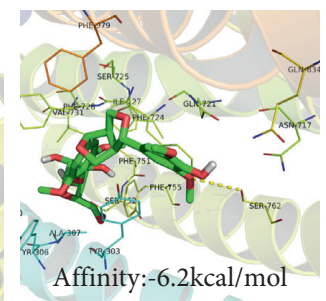

(A10)

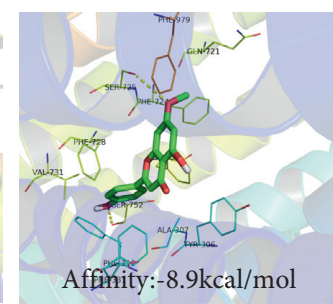

(A3)

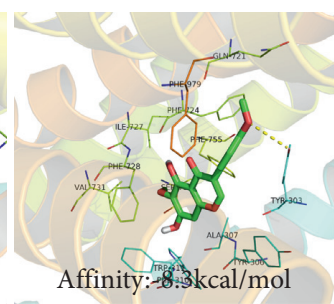

(A7)

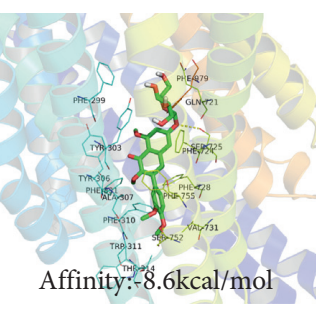

(A11)

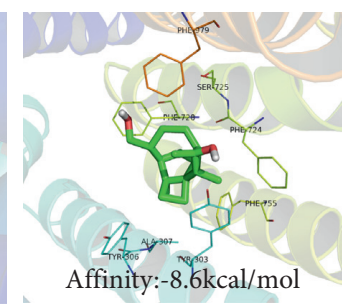

(A4)

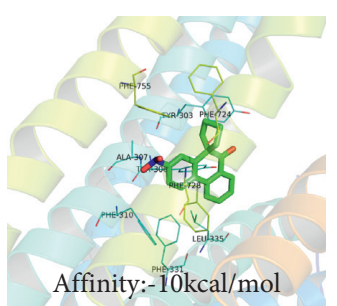

(A8)

(a)

Figure 1: Continued. 


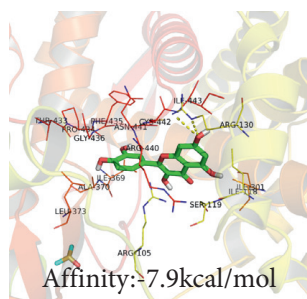

(B1)

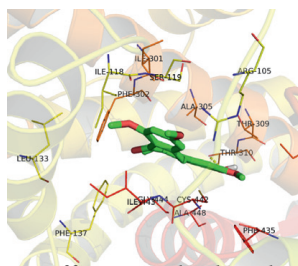

Affinity:-5.2kcal $/ \mathrm{mol}$

(B5)

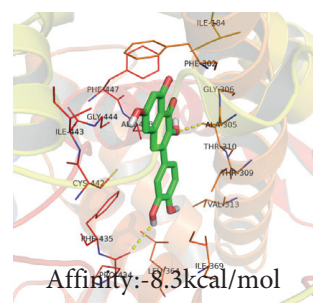

(B9)

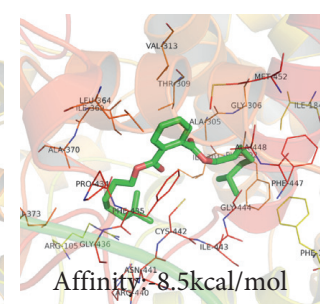

(B2)

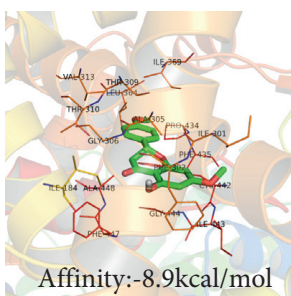

(B6)

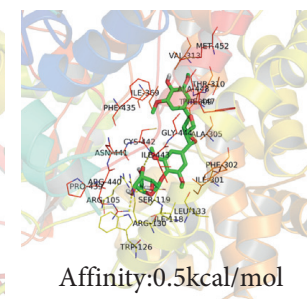

(B10)

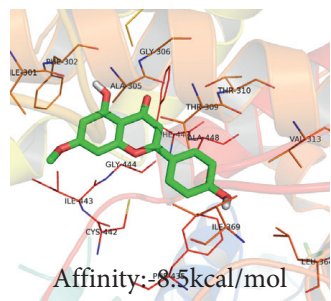

(B3)

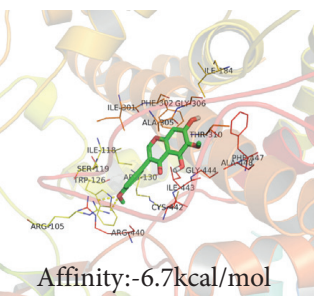

(B7)

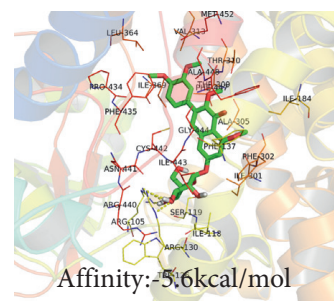

(B11)

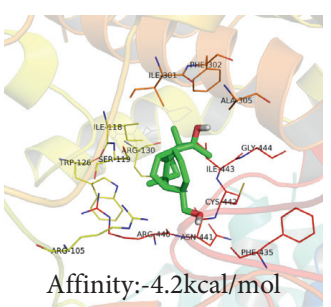

(B4)

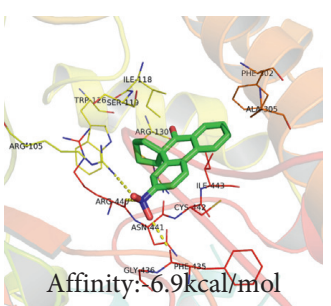

(B8)

(b)

Figure 1: The 11 active components of CPB docked with P-gp and CYP3A4 molecules. (a) P-gp. (b) CYP3A4. 1, quercetin; 2, Diop; 3, genkwanin; 4; patchoulan 1,12-diol; 5, pachypodol; 6, 5-hydroxy-7,4'-dimethoxyflavanone; 7, irisolidone; 8 , phenanthrone; 9 , quercetin 7O- $\beta$-D-glucoside; 10 , acanthoside $\mathrm{B} ; 11,3,23$-dihydroxy-12-oleanen-28-oic acid. $A 1, A 3, A 5, A 7, A 9, A 10, A 11, B 1, B 5, B 7, B 8, B 9, B 10$, and $B 11$ are hydrogen bond links and short-range van der Waals forces or $\pi$ interaction forces. $A 2, A 4, A 6, A 8, B 2, B 3, B 4$, and $B 6$ are short-range van der Waals forces or $\pi$ interaction force connections.

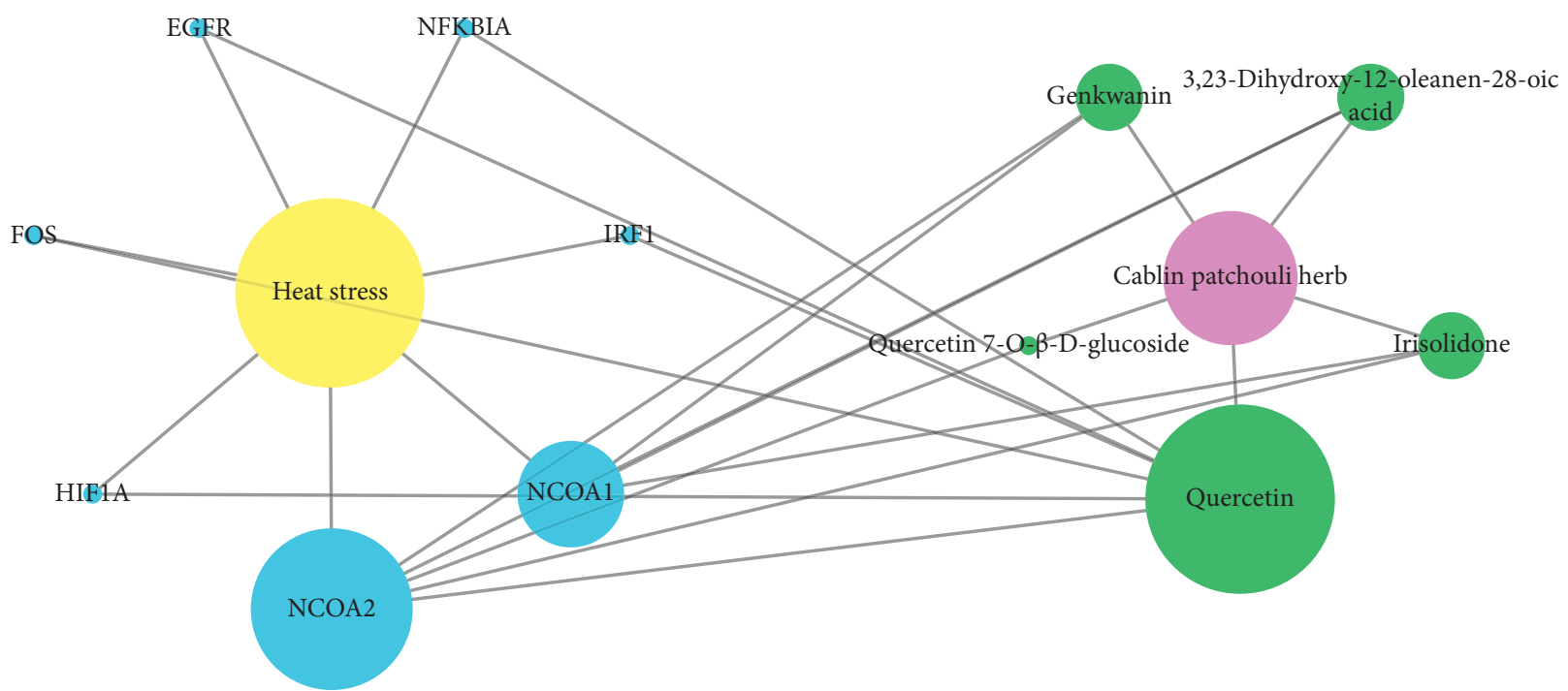

FIGURE 2: Drug-component-target-disease network. Blue represents targets, green represents drug molecules, magenta represents the drug, and yellow represents the disease. The size of each node represents its degree value: the larger the node is, the greater the degree value is. 


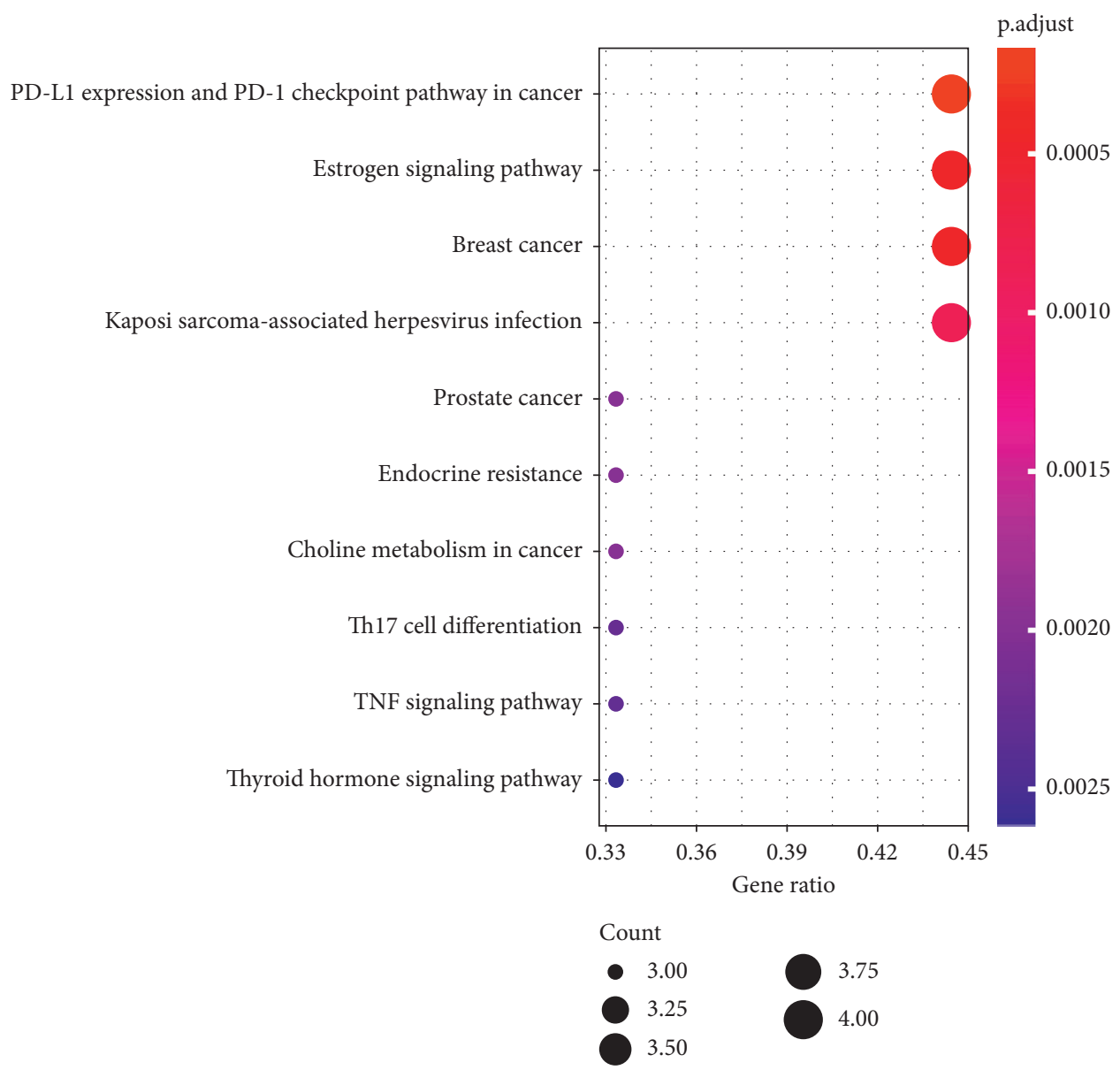

FIGURE 3: KEGG enrichment of CPB targets.

PD-L2 (B7-DC) represent the current main research directions with respect to immune checkpoint molecules [44]. The expression of PD-L1 is induced by a variety of proinflammatory factors (including type I and type II IFN- $\gamma$, IL$1 \beta$, TNF- $\alpha$, LPS, and so on) $[45,46]$. Proinflammatory factors are indispensable factors in the process of tumor formation and the main factors that cause fever in the human body. In conclusion, our research found that $\mathrm{CPB}$ may exert a certain regulatory effect on PD-L1 expression and the PD-1 checkpoint in cancer pathway.

Estrogen is a female steroid compound secreted by organs such as the ovaries and placenta. There are mainly three forms: estrone, estradiol, and estriol [47]. Previous studies have shown that estrogen can not only regulate reproductive behavior through estrogen receptors but also exert an important effect on the central nervous system [48]. Although the specific mechanism remains unclear, studies have shown that estrogen has a huge effect on body temperature regulation. The evidence includes the following: estrogen or related estrogen receptor ligand therapy can lower core temperature [49]; compared with ovariectomized rats that received estrogen, the temperature of skin vasodilation in ovariectomized rats was $4^{\circ}$ lower [50]; the state of estrogen deficiency will increase the sensitivity of the thermal defense pathway, leading to the activation of heat loss effect factors at lower ambient temperatures [51].
3.6. PPI Network. As the agents of cell activity and function, proteins do not exist independently. The interactions between proteins play important roles in each stage of life and maintain the steady state of the internal environment. To analyze the interaction modes of the CPB target proteins and the targets of the two signaling pathways enriched by the KEGG analysis, the cancer and estrogen signaling pathways, a PPI network of the CPB target proteins was constructed, as shown in Figure 4.

Among them, EGFR, NCOA1, and FOS are involved in two pathways. Epidermal growth factor receptor (EGFR) is a transmembrane glycoprotein that is one of the four members of the ErbB family of tyrosine kinase receptors. The activation of EGFR leads to the autophosphorylation of receptor tyrosine kinase, which triggers a series of downstream signaling pathways and participates in the regulation of cell proliferation, differentiation, and survival. EGFR is abnormally activated through various mechanisms, such as receptor overexpression, mutation, ligand-dependent receptor dimerization, and ligand-independent activation, and is associated with the occurrence of various human cancers. EGFR inhibition is one of the key targets of cancer chemotherapy [52]. Nuclear receptor coactivators (NCOAs) are multifunctional transcriptional coregulators of a growing number of signal-activated transcription factors. The members of the p160 family (NCOA1/2) are increasingly 


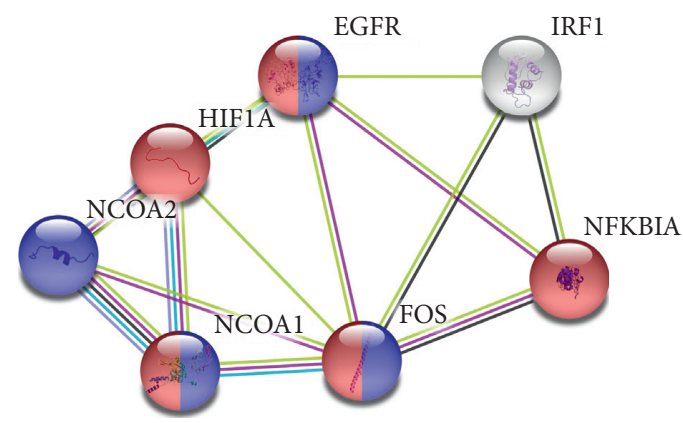

Figure 4: CPB target PPI network. Red indicates target proteins involved in cancer-related pathways; blue indicates target proteins involved in estrogen signaling.

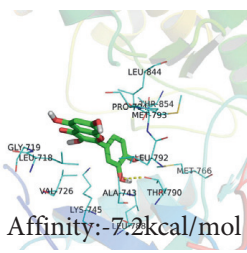

(A1)

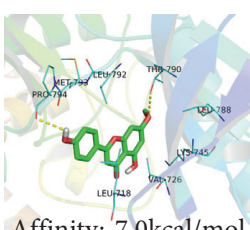

(B1)

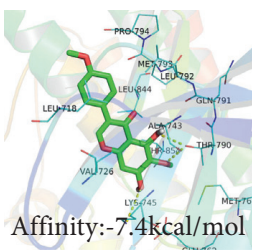

(C1)

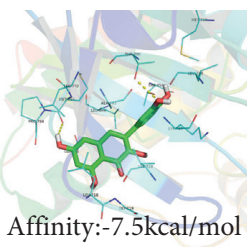

(D1)

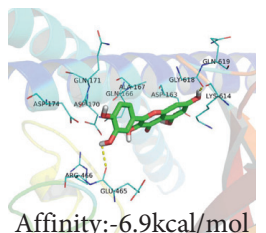

(A2)

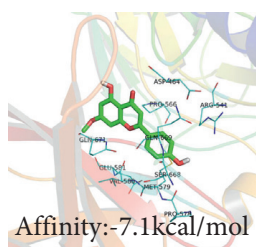

(B2)

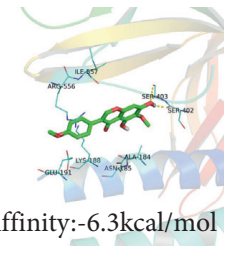

(C2)

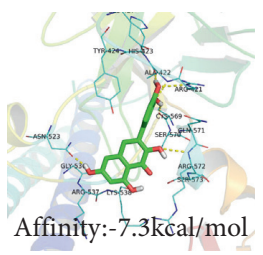

(D2)

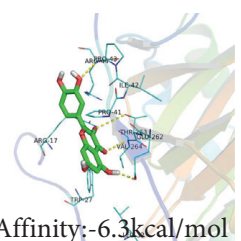

(A3)

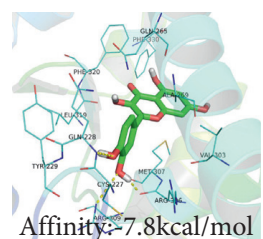

(A4)

(a)

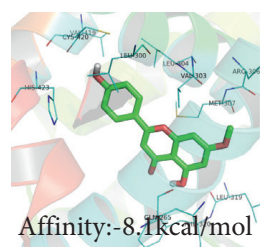

(B4)

(B3)

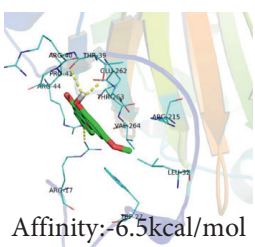

(b)

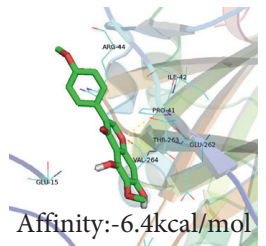

(C3)

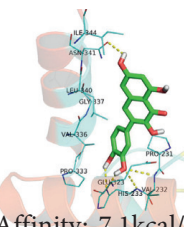

Affinity:-7.1 kcal $/ \mathrm{mol}$

(D3)

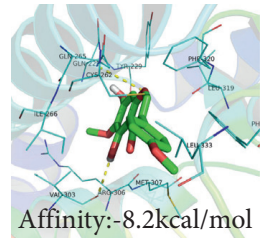

(C4)

(c)

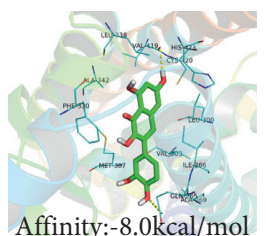

(D4)

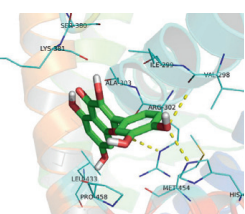

Affinity:-6.7kcal $/ \mathrm{mol}$

(A5)

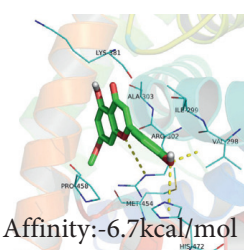

(B5)

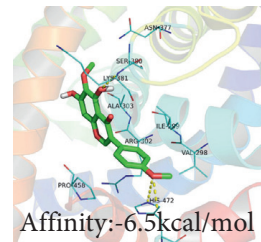

(C5)

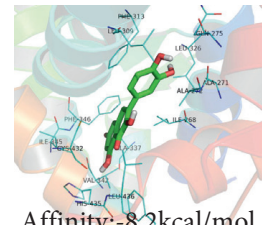

(D5)

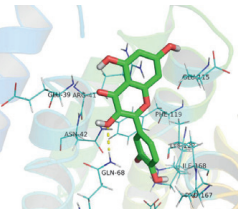

Affinity:-5.2 $\mathrm{kcal} / \mathrm{mol}$

(A6)

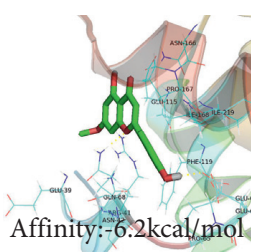

(B6)

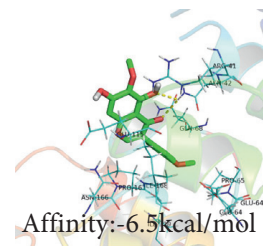

(C6)

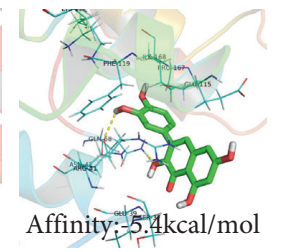

(D6)

(d)

Figure 5: Continued. 


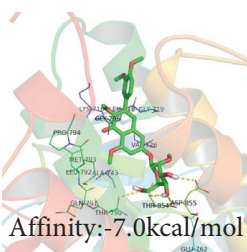

(E1)

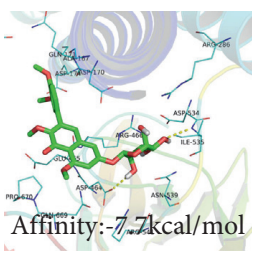

(E2)

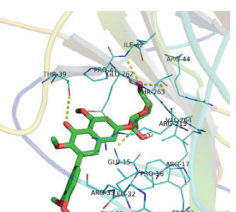

Affinity:-6.9 $\mathrm{kcal} / \mathrm{mol}$

(E3)

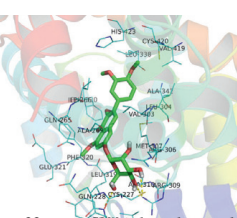

Affinity:- -7

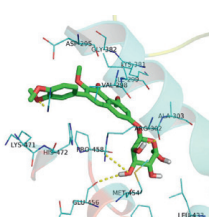

Affinity:-6.8kcal/mol

(E5)

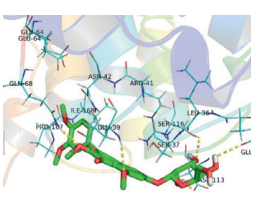

Affinity:-5.7kcal/mol

(E6)

(e)

FIGURE 5: Docking results of CPB components and target molecules. (a) Quercetin. (b) Genkwanin. (c) Irisolidone. (d) Quercetin 7-O- $\beta$-Dglucoside. (e) 3,23-Dihydroxy-12-oleanen-28-oic acid. 1, EGFR; 2, FOS; 3, HIF1A; 4; NCOA1; 5, NCOA2; 6, NFKBIA. A1, $A 2, A 3$, $A 4$, $A 5$, $A 6, B 1, B 3, B 5, B 6, C 1, C 2, C 3, C 4, C 5, C 6, D 1, D 2, D 3, D 4, D 6, E 1, E 2, E 3, E 4, E 5$, and $E 6$ are hydrogen bond links and short-range van der Waals forces or $\pi$ interaction forces. $B 2, B 4$, and $D 5$ are short-range van der Waals forces or $\pi$ interaction force connections.

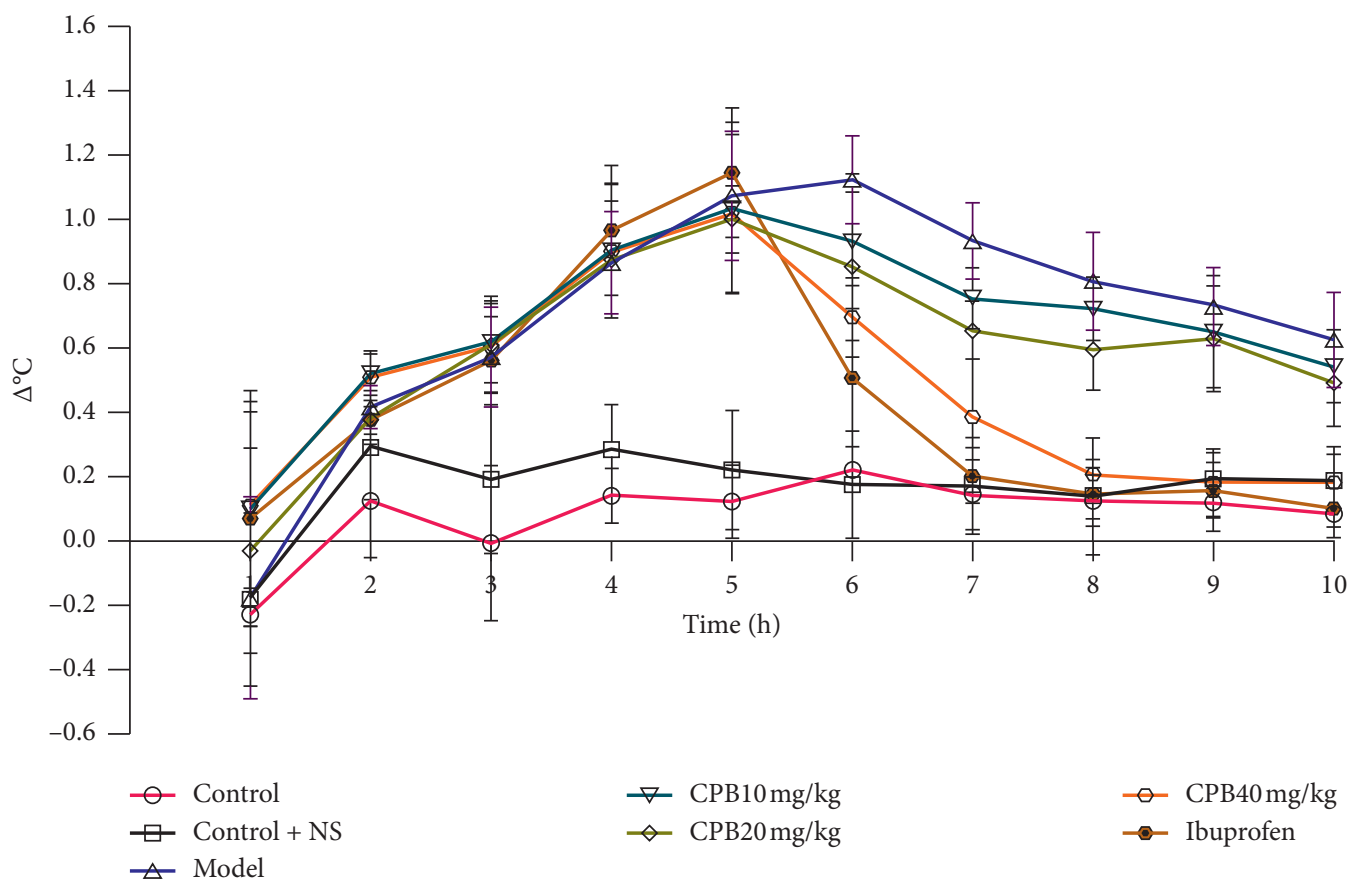

(a)

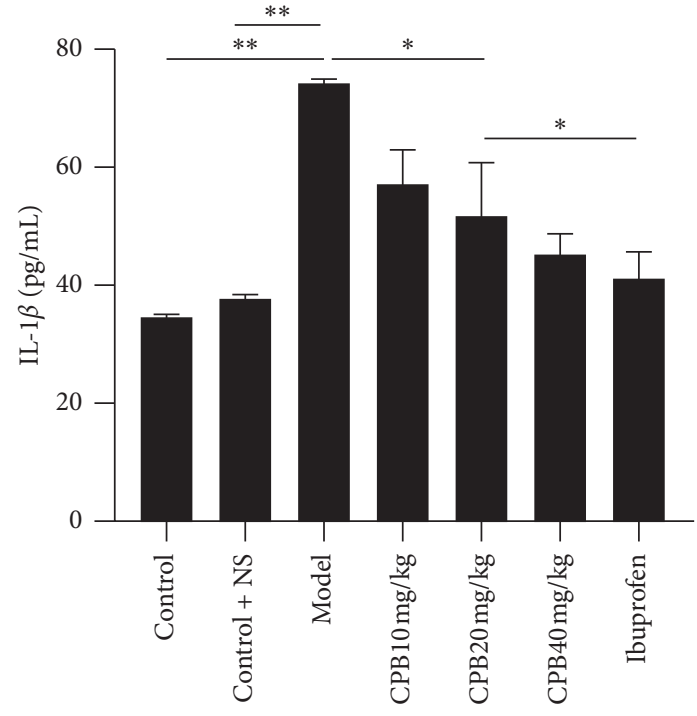

(b)

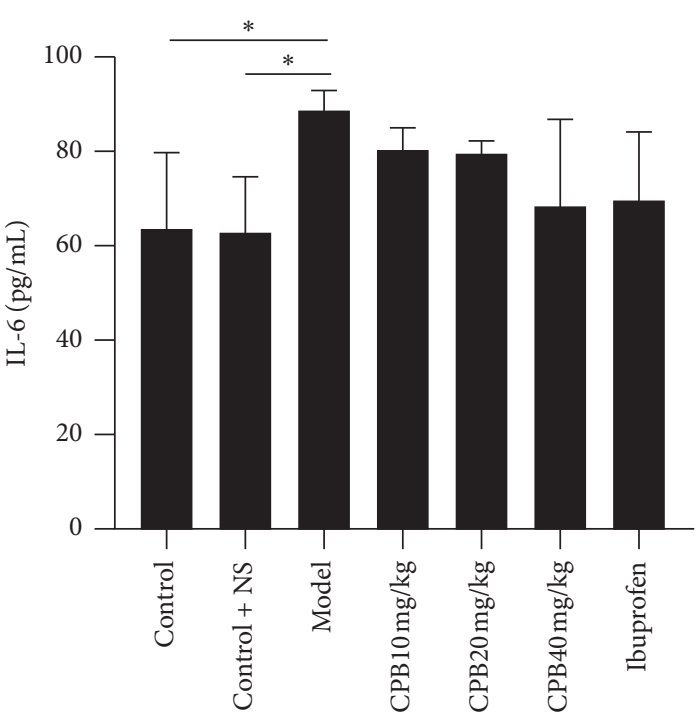

(c)

FIGURE 6: Continued. 


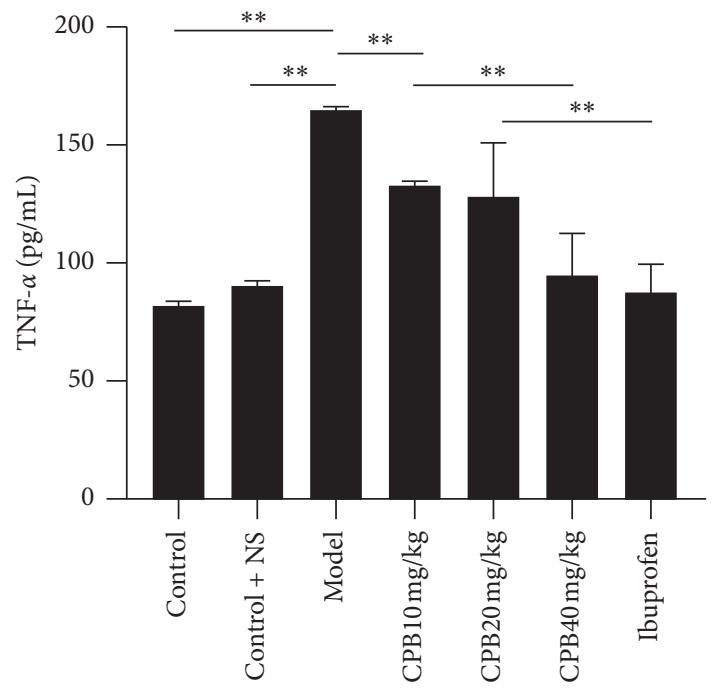

(d)

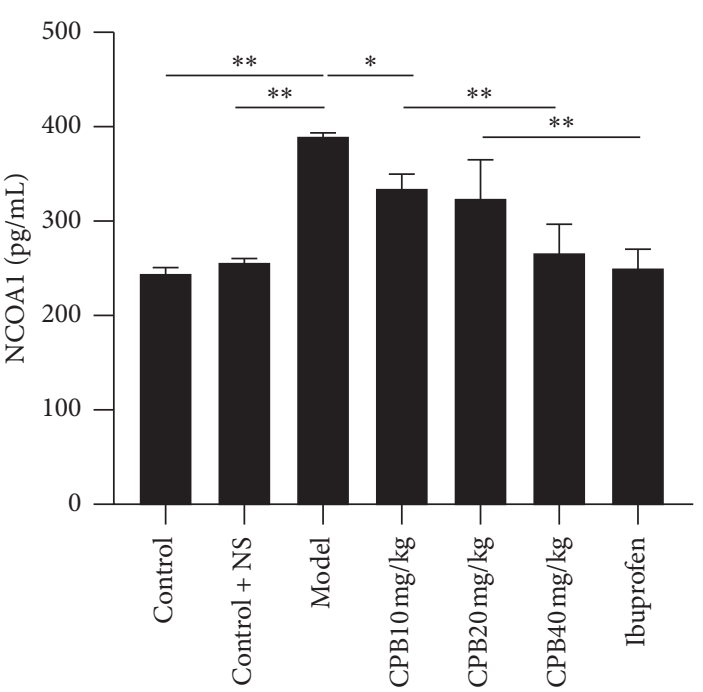

(e)

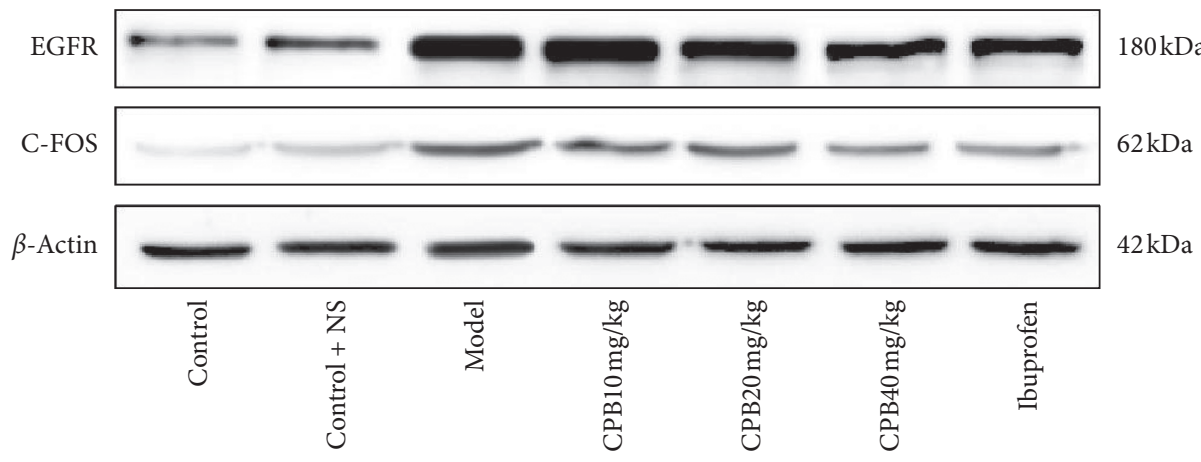

(f)

Figure 6: The effect of CPB on rats. (a) CPB on the body temperature of rats. (b) $\mathrm{CPB}$ on the amount of IL- $1 \beta$ in rat serum. (c) $\mathrm{CPB}$ on the amount of IL-6 in rat serum. (d) CPB on the amount of TNF- $\alpha$ in rat serum. (e) CPB on the amount of NCOAl in rat serum. (f) CPB on RGFR and C-FOS protein.

recognized as essential and nonredundant players in a number of physiological processes [53]. This family has been shown to be related to cancer [54] and inflammation and metabolism [53]. FOS is a nuclear phosphoprotein encoded by mature mRNA transcribed from the c-fos gene and is closely related to seizures [55] and cancer [56]. Hypoxiainducible factor 1-alpha (HIF1A) is an oxygen-dependent transcriptional activator that plays key roles in tumor angiogenesis and mammalian development [57]. Finally, NFKBIA encodes inhibitors of nuclear factor $-\kappa \mathrm{B}(\mathrm{NF}-\kappa \mathrm{B})$ that regulate the translation of genes involved in inflammatory and immune reactions $[58,59]$. In conclusion, our results demonstrate that EGFR, NCOA1, FOS, HIF1A, NFKBIA, and NCOA2 may be the main targets of CPB.

3.7. Molecular Docking Verification. In the above, we showed that quercetin, genkwanin, irisolidone, 3,23-dihydroxy-12-oleanen-28-oic acid, and quercetin 7-O- $\beta$-D-glucoside are the main components of CPB. EGFR, NCOA1, FOS, HIF1A, NFKBIA, and NCOA2 are the main target proteins, which we analyzed to more clearly verify whether the components and the targets can actually interact. We continued to use molecular docking technology for verification, and the results are shown in Figure 5. In short, the molecular docking verification of the 5 main components of $\mathrm{CPB}$ and the 6 main target proteins was successful.

3.8. Animal Experiment Verification. To further prove the availability of $C P B$, a rat model of fever was used to study $\mathrm{CPB}$ intragastric treatment. As shown in Figure 6(a), CPB at a concentration of $40 \mathrm{mg} / \mathrm{kg}$ showed a significant antipyretic effect after treatment. At the same time, we also measured the values of IL- $1 \beta$, IL- 6 , TNF- $\alpha$, and NCOA1 in the blood. As shown in Figures 6(b), 6(d), and 6(e), CPB can reduce the levels of IL- $1 \beta, \mathrm{TNF}-\alpha$, and NCOA1 in rat serum. In Figure 6(c), the amount of IL-6 also seems to decrease, although it is not statistically significant. To further verify the conclusions we have reached, we used the protein expression in the rat hypothalamus to perform western blot verification. As shown in Figure 6(f), the expression of EGFR and C-FOS proteins in rats increased after modeling, but after $\mathrm{CPB}$ treatment, the expression of EGFR and C-FOS proteins decreased. In short, all animal experiments have successfully verified the conclusions we have reached. 


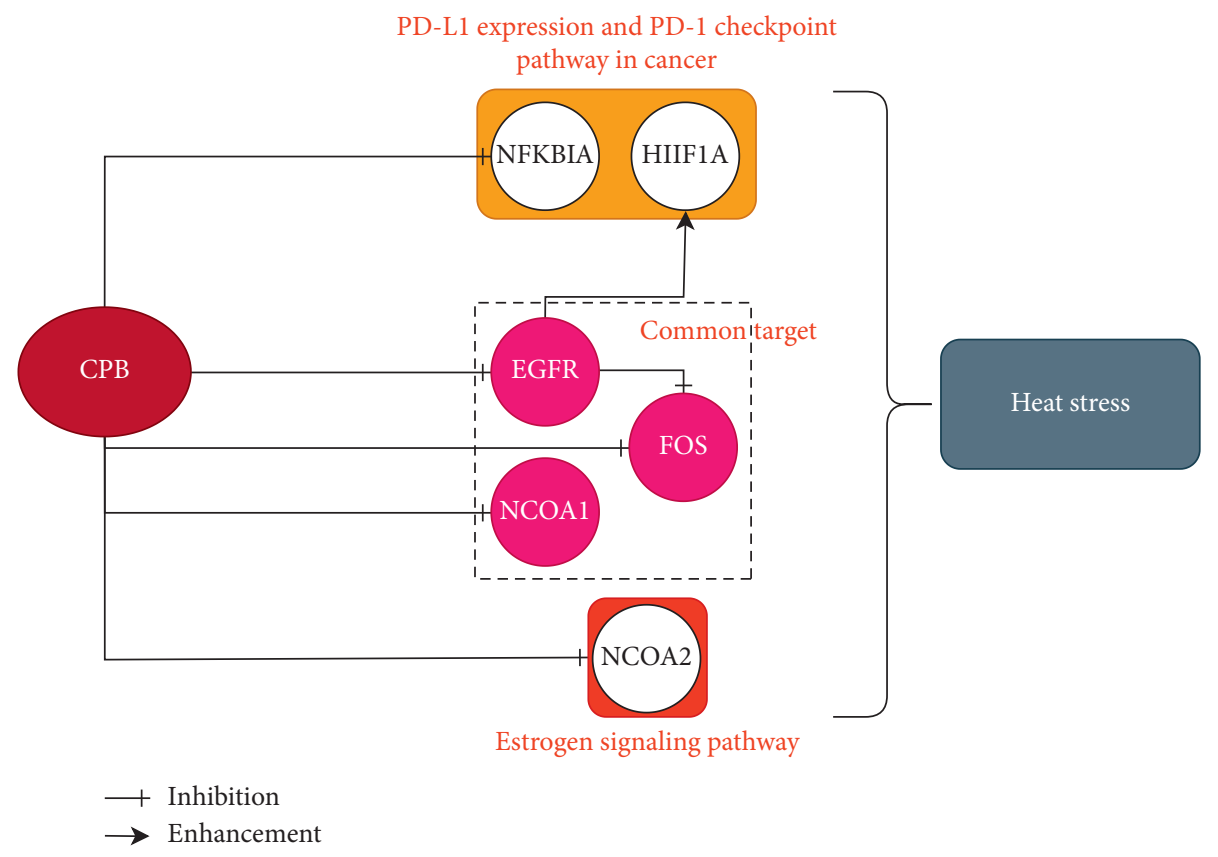

FIgURE 7: The target map of CPB for the treatment of heat stress.

\section{Discussion}

This study used network pharmacology combined with molecular docking. First, we collected the components and targets of $\mathrm{CPB}$ and screened them. Then, the ingredients were used for molecular docking with P-gp and CYP3A4 to initially verify that the active ingredients can be absorbed by the body. Then, we obtained genes which are differentially expressed during heat stress from the GEO database, constructed a drug-component-target-disease network, and further identified quercetin, genkwanin, irisolidone, 3,23-dihydroxy-12-oleanen-28-oic acid, and quercetin 7-O- $\beta$-D-glucoside as the main ingredients of CPB. We also constructed KEGG and PPI networks based on target information and proved that EGFR, NCOA1, FOS, HIF1A, NFKBIA, and NCOA2 are the main targets. Finally, the 5 active compounds were used to verify molecular docking with the 6 main targets.

To enhance the level of evidence for CPB in the treatment of heat stress, we continued to use febrile rats for experimental verification. We first proved that $\mathrm{CPB}$ can reduce the body temperature of febrile rats. The values of IL$1 \beta$, TNF- $\alpha$, and NCOA1 in the blood of rats also decreased. Western blot detection also found that EGFR and C-FOS proteins in the hypothalamus were reduced. We have also produced a map of the targets of CPB, as shown in Figure 7.

In conclusion, our study shows that the main components of $\mathrm{CPB}$ as a treatment for heat stress are quercetin, genkwanin, irisolidone, 3,23-dihydroxy-12-oleanen-28-oic acid, and quercetin 7-O- $\beta$-D-glucoside. Its main targets are EGFR, NCOA1, FOS, HIF1A, NFKBIA, and NCOA2. Our study is the first network pharmacology study and experimental verification of the use of the traditional Chinese medicine $\mathrm{CPB}$ to treat heat stress; thus, this research has pioneering significance.

\section{Data Availability}

The research data used to support the findings of this study are included within the supplementary information files.

\section{Conflicts of Interest}

The authors declare that they have no conflicts of interest.

\section{Authors' Contributions}

Yan $\mathrm{Xu}$ and Lizhong Ding contributed equally to this study.

\section{Acknowledgments}

This study was supported by the National Key Research and Development Program of China (2017YFC1703202), the Jilin Scientific and Technological of Chinese Medicine Program (2019023), the Inheritance and Innovation of Chinese Medicine "Millions of Standouts" Project (the Project of Qihuang), the Inheritance Workroom of the Chinese Medicine Master Wang Lie, and the Jilin Scientific and Technological Program of Sanitation and Population Control (2018J106).

\section{Supplementary Materials}

Supplementary Table 1: 1789 heat stress differentially expressed genes. Supplementary Table 2: the CPB components and targets after correction. (Supplementary Materials) 


\section{References}

[1] J. C. Semenza, C. H. Rubin, K. H. Falter et al., "Heat-related deaths during the July 1995 heat wave in Chicago," New England Journal of Medicine, vol. 335, no. 2, pp. 84-90, 1996.

[2] J.-M. Robine, S. L. K. Cheung, S. Le Roy et al., "Death toll exceeded 70,000 in Europe during the summer of 2003," Comptes Rendus Biologies, vol. 331, no. 2, pp. 171-178, 2008.

[3] D. Barriopedro, E. M. Fischer, J. Luterbacher, R. M. Trigo, and R. García-Herrera, "The hot summer of 2010: redrawing the temperature record map of Europe," Science, vol. 332, no. 6026, pp. 220-224, 2011.

[4] C. W. Mangus and T. L. Canares, "Heat-related illness in children in an era of extreme temperatures," Pediatrics in Review, vol. 40, no. 3, pp. 97-107, 2019.

[5] D. J. Casa, L. E. Armstrong, R. Carter, R. Lopez, B. McDermott, and K. Scriber, "Historical perspectives on medical care for heat stroke, Part 1: ancient times through the nineteenth century," Athletic Training \& Sports Health Care, vol. 2, no. 3, pp. 132-138, 2010.

[6] C. R. Gomez, "Disorders of body temperature," Neurologic Aspects of Systemic Disease Part II, vol. 120, pp. 947-957, 2014.

[7] A. Bouchama and J. P. Knochel, "Heat stroke," New England Journal of Medicine, vol. 346, no. 25, pp. 1978-1988, 2002.

[8] J. R. Lepock, "Cellular effects of hyperthermia: relevance to the minimum dose for thermal damage," International Journal of Hyperthermia, vol. 19, no. 3, pp. 252-266, 2003.

[9] D. Kültz, "Molecular and evolutionary basis of the cellular stress response," Annual Review of Physiology, vol. 67, no. 1, pp. 225-257, 2005.

[10] K. Richter, M. Haslbeck, and J. Buchner, "The heat shock response: life on the verge of death," Molecular Cell, vol. 40, no. 2, pp. 253-266, 2010.

[11] L. fusen, "Scraping and huoxiang zhengqi water for 48 cases of yin and heat," Chinese Folk Remedies, vol. 12, pp. 23-24, 1999.

[12] X. Li, "Observation on the curative effect of Huoxiangzhengqi water rubbing the back to treat children with high fever (Chinese)," Contemporary Clinical Medicine, vol. 28, no. 3, pp. 1387-1388, 2015.

[13] L. Y. Gu Fang and C. Rinan, "Huoxiang zhengqi water in treating 18 cases of children's heat rash (Chinese)," Chinese Practical Journal of Rural Doctors, vol. 9, 2005.

[14] L. Chaobin and G. Wanzhou, "Discussion on huoxiang zhengqi water in treating heat stroke(Chinese)," Shaanxi Traditional Chinese Medicine, vol. 31, no. 12, p. 1668, 2010.

[15] H. J. Zhao, L. P. Guo, F. W. Yang et al., "[Huoxiang Zhengqi formulas for treatment of gastrointestinal type cold:a systematic review and Meta-analysis]," China Journal of Chinese Materia Medica, vol. 42, no. 8, pp. 1495-1499, 2017.

[16] Y. Liu, W. Liu, Q. X. Peng, J. L. Peng, L. Z. Yu, and J. L. Hu, "Protective effect of huoxiang zhengqi oral liquid on intestinal mucosal mechanical barrier of rats with postinfectious irritable bowel syndrome induced by acetic Acid," Evidencebased Complementary and Alternative Medicine, vol. 2014, Article ID 218383, 10 pages, 2014.

[17] Z. Xu, "Modernization: one step at a time," Nature, vol. 480, no. 7378, pp. S90-S92, 2011.

[18] S. Li and B. Zhang, "Traditional Chinese medicine network pharmacology: theory, methodology and application," Chinese Journal of Natural Medicines, vol. 11, no. 2, pp. 110-120, 2013.

[19] S. Li, Z. Q. Zhang, L. J. Wu, X. G. Zhang, Y. D. Li, and Y. Y. Wang, "Understanding ZHENG in traditional Chinese medicine in the context of neuro-endocrine-immune network," IET Systems Biology, vol. 1, no. 1, pp. 51-60, 2007.

[20] S. Li, "Network systems underlying traditional Chinese medicine syndrome and herb formula," Current Bioinformatics, vol. 4, no. 3, 2009.

[21] S. Li, "[Network target: a starting point for traditional Chinese medicine network pharmacology]," China Journal of Chinese Materia Medica, vol. 36, no. 15, pp. 2017-2020, 2011.

[22] S. Li, B. Zhang, and N. Zhang, "Network target for screening synergistic drug combinations with application to traditional Chinese medicine," BMC Systems Biology, vol. 5, no. 1, p. S10, 2011.

[23] H. Li, L. Zhao, B. Zhang et al., "A network pharmacology approach to determine active compounds and action mechanisms of ge-gen-qin-lian decoction for treatment of type 2 diabetes," Evidence-based Complementary and Alternative Medicine, vol. 2014, Article ID 495840, 12 pages, 2014.

[24] J. Ru, P. Li, J. Wang et al., "TCMSP: a database of systems pharmacology for drug discovery from herbal medicines," Journal of Cheminformatics, vol. 6, p. 13, 2014.

[25] G. Yu, L.-G. Wang, Y. Han, and Q.-Y. He, "clusterProfiler: an $\mathrm{R}$ package for comparing biological themes among gene clusters," OMICS: A Journal of Integrative Biology, vol. 16, no. 5, pp. 284-287, 2012.

[26] Z. F. Wang, Y. Q. Hu, Q. G. Wu, and R. Zhang, "Virtual screening of potential anti-fatigue mechanism of polygonati rhizoma based on network pharmacology," Combinatorial Chemistry \& High Throughput Screening, vol. 22, no. 9, pp. 612-624, 2019.

[27] M. Chuan, P. Cheng, L. Xinrui, X. liang, and Z. Qinmei, "Research progress on chemical constituents and pharmacological activities of patchouli (Chinese)," Journal of Chengdu University of Traditional Chinese Medicine, vol. 43, no. 1, pp. 72-80, 2020.

[28] M. Pertino, J. A. Rodríguez, C. Theoduloz, I. Razmilic, and G. Schmeda-Hirschmann, "Gastroprotective activity and cytotoxic effect of cyperenoic acid derivatives," The Journal of Pharmacy and Pharmacology, vol. 58, no. 11, 2006.

[29] Q. M. Zhou, C. Peng, X. H. Li, L. Guo, L. Xiong, and D. S. Lin, "[Study on constituents of the aerial parts of Pogostemon cablin]," Journal of Chinese Medicinal Materials, vol. 36, no. 6, pp. 915-918, 2013.

[30] M. Swamy and U. Sinniah, "A comprehensive review on the phytochemical constituents and pharmacological activities of pogostemon cablin benth.: an aromatic medicinal plant of industrial importance," Molecules: A Journal of Synthetic Chemistry and Natural Product Chemistry, vol. 20, no. 5, 2015.

[31] S. N. Ruan, Y. Lu, and D. F. Chen, "[Anti-complementary constituents of Pogostemon cablin]," China Journal of Chinese Materia Medica, vol. 38, no. 13, pp. 2129-2135, 2013.

[32] M. Ramar, M. G. Rao, S. Veerappan, and B. Pemaiah, "Pogostemon hirsutus oil, rich in abietane diterpenes," Natural Product Communications, vol. 8, no. 12, 2013.

[33] M. Miyazawa, Y. Okuno, S. Nakamura, and H. Kosaka, "Antimutagenic activity of flavonoids from Pogostemon cablin," Journal of Agricultural and Food Chemistry, vol. 48, no. 3, 2000.

[34] V. Kumar, V. Shriram, R. Bhagat, T. Khare, S. Kapse, and N. Kadoo, "Phytochemical profile, anti-oxidant, anti-inflammatory, and anti-proliferative activities of Pogostemon deccanensis essential oils," 3 Biotech, vol. 9, no. 1, p. 31, 2019.

[35] Y. Yao, X. Zhang, Z. Wang et al., "Deciphering the combination principles of Traditional Chinese Medicine from a systems pharmacology perspective based on Ma-huang 
Decoction," Journal of Ethnopharmacology, vol. 150, no. 2, pp. 619-638, 2013.

[36] M. Varma, Y. Ashokraj, C. S. Dey, and R. Panchagnula, "Pglycoprotein inhibitors and their screening: a perspective from bioavailability enhancement," Pharmacological Research, vol. 48, no. 4, pp. 347-359, 2003.

[37] F. P. Guengerich, "Cytochrome P-450 3A4: regulation and role in drug metabolism," Annual Review of Pharmacology and Toxicology, vol. 39, no. 1, pp. 1-17, 1999.

[38] Y. S. Kumar, D. Adukondalu, D. Sathish et al., "P-Glycoprotein- and cytochrome P-450-mediated herbal drug interactions," Drug Metabolism And Drug Interactions, vol. 25, no. 1-4, pp. 3-16, 2010.

[39] Y. Wei, D. Zhou, J. Peng, L. Pan, and K. Tu, "Hot air treatment induces disease resistance through activating the phenylpropanoid metabolism in cherry tomato fruit," Journal of Agricultural and Food Chemistry, vol. 65, no. 36, pp. 80038010, 2017.

[40] D. Xu, M. Hu, Y. Wang, and Y. Cui, "Antioxidant activities of quercetin and its complexes for medicinal application," Molecules: A Journal of Synthetic Chemistry and Natural Product Chemistry, vol. 24, no. 6, 2019.

[41] T. Sjöblom, S. Jones, L. D. Wood et al., "The consensus coding sequences of human breast and colorectal cancers," Science, vol. 314, no. 5797, pp. 268-274, 2006.

[42] T. R. Mosmann and S. Sad, "The expanding universe of T-cell subsets: Th1, Th2 and more," Immunology Today, vol. 17, no. 3, pp. 138-146, 1996.

[43] W. Zou, "Immunosuppressive networks in the tumour environment and their therapeutic relevance," Nature Reviews Cancer, vol. 5, no. 4, pp. 263-274, 2005.

[44] B. T. Fife and J. A. Bluestone, "Control of peripheral T-cell tolerance and autoimmunity via the CTLA-4 and PD-1 pathways," Immunological Reviews, vol. 224, no. 1, pp. 166-182, 2008.

[45] A. Kondo, T. Yamashita, H. Tamura et al., "Interferon- $\gamma$ and tumor necrosis factor- $\alpha$ induce an immunoinhibitory molecule, B7-H1, via nuclear factor- $\kappa \mathrm{B}$ activation in blasts in myelodysplastic syndromes," Blood, vol. 116, no. 7, pp. 1124-1131, 2010.

[46] M. Sznol and L. Chen, "Antagonist antibodies to PD-1 and B7-H1 (PD-L1) in the treatment of advanced human cancer," Clinical Cancer Research, vol. 19, no. 5, pp. 1021-1034, 2013.

[47] B. Li, J. Zhao, J. Lv et al., "Additive antidepressant-like effects of fasting with imipramine via modulation of 5-HT2 receptors in the mice," Progress in Neuro-Psychopharmacology and Biological Psychiatry, vol. 48, pp. 199-206, 2014.

[48] J.-A. Gustafsson, "What pharmacologists can learn from recent advances in estrogen signalling," Trends in Pharmacological Sciences, vol. 24, no. 9, pp. 479-485, 2003.

[49] T. A. Roepke, M. A. Bosch, E. A. Rick et al., "Contribution of a membrane estrogen receptor to the estrogenic regulation of body temperature and energy homeostasis," Endocrinology, vol. 151, no. 10, pp. 4926-4937, 2010.

[50] P. A. Dacks and N. E. Rance, "Effects of estradiol on the thermoneutral zone and core temperature in ovariectomized rats," Endocrinology, vol. 151, no. 3, pp. 1187-1193, 2010.

[51] N. E. Rance, P. A. Dacks, M. A. Mittelman-Smith, A. A. Romanovsky, and S. J. Krajewski-Hall, "Modulation of body temperature and LH secretion by hypothalamic KNDy (kisspeptin, neurokinin B and dynorphin) neurons: a novel hypothesis on the mechanism of hot flushes," Frontiers in Neuroendocrinology, vol. 34, no. 3, pp. 211-227, 2013.
[52] D. Singh, B. Kumar Attri, R. Kaur Gill, and J. Bariwal, "Review on EGFR inhibitors: critical updates," Mini-Reviews in $\mathrm{Me}$ dicinal Chemistry, vol. 16, no. 14, pp. 1134-1166, 2016.

[53] D. A. Rollins, M. Coppo, and I. Rogatsky, "Minireview: nuclear receptor coregulators of the p160 family: insights into inflammation and metabolism," Molecular Endocrinology, vol. 29, no. 4, pp. 502-517, 2015.

[54] S. A. Oñate, S. Y. Tsai, M. J. Tsai, and B. W. O’Malley, “Sequence and characterization of a coactivator for the steroid hormone receptor superfamily," Science (New York, N.Y.), vol. 270, no. 5240, pp. 1354-1357, 1995.

[55] D. G. Herrera and H. A. Robertson, "Activation of c-fos in the brain,” Progress in Neurobiology, vol. 50, no. 2-3, pp. 83-107, 1996.

[56] A. Krishna, M. Bhatt, V. Singh et al., "Differential expression of $\mathrm{c}$-fos proto-oncogene in normal oral mucosa versus squamous cell carcinoma," Asian Pacific Journal of Cancer Prevention: APJCP, vol. 19, no. 3, pp. 867-874, 2018.

[57] J.-W. Lee, S.-H. Bae, J.-W. Jeong, S.-H. Kim, and K.-W. Kim, "Hypoxia-inducible factor (HIF-1) $\alpha$ : its protein stability and biological functions," Experimental \& Molecular Medicine, vol. 36, no. 1, pp. 1-12, 2004.

[58] G. L. Zhang, Y. F. Zou, X. L. Feng et al., "Association of the NFKBIA gene polymorphisms with susceptibility to autoimmune and inflammatory diseases: a meta-analysis," Inflammation research: official journal of the European Histamine Research Society, vol. 60, no. 1, pp. 11-18, 2011.

[59] M. Zhang, J. Huang, X. Tan et al., "Common polymorphisms in the NFKBIA gene and cancer susceptibility: a meta-analysis," Medical Science Monitor, vol. 21, pp. 3186-3196, 2015. 